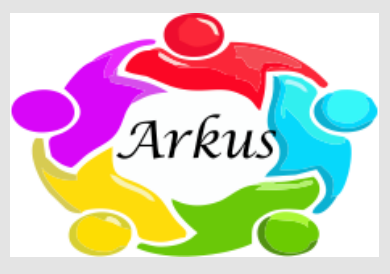

\title{
Bridging Coronary Physiology into Clinical Application of Acute Coronary
}

\section{Syndrome}

\section{Hardi Darmawan ${ }^{*}$}

${ }^{1}$ Department of Physiology, Faculty of Medicine, Sriwijaya University, Palembang, Indonesia

\section{A R T I C L E I N F O}

\section{Keywords:}

Physiology

Application

Acute coronary syndrome

\section{Corresponding author:}

Hardi Darmawan

E-mail address:

\section{hardidarmawan@gmail.com}

The author has reviewed and approved the final version of the manuscript.

https://doi.org/10.37275/arkus.v5i1.123

\begin{abstract}
A B S T R A C T
Normal arteries have three layers of structure, tunica intima, tunica media, and tunica adventitia. Intima tunica is the deepest layer of coronary arteries in which there are antithrombotic molecules such as heparin sulfate, thrombomodulin, and plasminogen activator. In addition, tunica intima also contains substances that regulate the contraction of tunica smooth muscle cell media, called nitric oxide (vasodilators) and prostacyclin (vasoconstrictors). Tunica intima and tunica media seem to be directly related to the atherosclerosis process. Meanwhile, the role of tunika adventisia is unknown. The accumulation of atherosclerotic lesions and hemodynamic stress factors and the degradation of extracellular matrix will cause susceptibility of atherosclerotic plaque fibrous capsules to rupture and form thrombus. Thrombus that occurs in the coronary condition causes acute coronary syndrome, characterized by typical symptoms such as chest pain depending on the thrombus formed. In studying acute coronary syndromes, of course it cannot be separated from understanding the physiology of coronary arteries and the process of atherosclerosis. Therefore, this article aims to briefly explain coronary physiology.
\end{abstract}

\section{Introduction}

The coronary arteries consist of the tunica intima, tunica media and tunica adventitia. The tunica intima is the innermost layer of the coronary arteries which contains antithrombotic molecules such as heparin sulfate, thrombomodulin, and plasminogen activator. In addition, the tunica intima also contains substances that regulate the contraction of smooth muscle cells in the tunica media, namely nitric oxide (a vasodilator) and prostacyclin (a vasoconstrictor). The tunica intima and tunica media appear to be directly related in the process of atherosclerosis. Meanwhile, the role of the tunica adventitia is not known for certain in atherosclerosis. ${ }^{1}$ Atherosclerosis is an inflammatory process that preceded endothelial dysfunction and accumulation of lipid oxidation mainly occurs in medium and large sized arteries such as the aorta, carotid arteries, coronary arteries and the arteries of the lower extremities. Atherosclerosis begins with the accumulation of extracellular lipids in the form of LDL (low density lipoprotein that penetrates the tunica intima and binds to the arterial intima proteoglycan matrix and then coalesces to form aggregates. This bond causes oxidized LDL. Oxidized LDL is immunogenic causing activation of endothelial cells, monocytes/macrophages). and T cells, which in turn leads to the recruitment of leukocytes. The initiation and development of atherosclerotic plaques generally 
requires a process that lasts for years with no symptoms experienced by the patient. Plaque complications such as calcification, rupture, hemorrhage and embolism can result in acute reduced blood flow or changes in blood flow. integrity of the walls of blood vessels so that they show different clinical symptoms in each organ system. Thrombosis is a complication of plaque that occurs in the myocardium which will cause acute coronary syndrome. In studying acute coronary syndrome, it is certainly not can be separated from understanding coronary vascular physiology and the process of atherosclerosis. ${ }^{2-3}$

\section{Pathogenesis}

The current acute coronary syndrome emphasizes thrombosis as an important mechanism of the transition from chronic to acute atherosclerotic disease. Plaque with a thin fibrous capsule, large lipid accumulation, abundant inflammatory cells, and relatively few smooth muscle cells are known to cause fatal coronary thrombosis. There are three causes of acute thrombosis caused by atherosclerotic plaque disruption. First, approximately two-thirds of acute myocardial infarctions result from rupture of the plaque's fibrous capsule. Apoptosis of macrophages produces thrombogenic microparticles that cause plaque disruption resulting in thrombosis. The second is the occurrence of superficial erosion of the plaque characterized by a large thrombus consisting of smooth muscle cells and very little inflammation. The third is a nodule of unknown calcification but often there is fibrin, osteoclasts between the calcifications. Atherosclerotic lesions with calcified nodules are common in males, and can be found in the middle of the right coronary artery or the left anterior descending coronary artery. Acute coronary syndrome is a spectrum of clinical conditions in a continuum associated with myocardial damage. Acute coronary syndromes are differentiated based on clinical, electrocardiogram, and laboratory findings, namely acute myocardial infarction with ST elevation (AMI-EST) and acute myocardial infarction without ST elevation (differentiated into unstable angina pectoris (APTS) and AMI-NEST. APTS and IMA-NEST consist of anti-ischemic drugs with the aim of restoring the balance between myocardial oxygen supply and demand, anti-thrombotic therapy to prevent expansion and facilitate the destruction of partially closed coronary.Nitrates relieve angina through venodilation by decreasing myocardial oxygen demand with flow. venous return to the heart, thereby reducing ventricular wall tension and preventing vasospasm through coronary venodilation.Other anti-ischemic therapies are beta blockers and CCBs. Anti-thrombotic therapy includes antiplatelet and anticoagulation drugs aimed at preventing the progression of partially closed intracoronary thrombus. with APTS and IMANEST, arteries affected in IMA-EST, completely occluded, to reduce myocardial damage, the main focus of treatment is to achieve reperfusion very rapidly, either mechanically with percutaneous revascularization or medically with fibrinolytics. ${ }^{4-9}$

\section{Conclusion}

Normal arteries have three layers of structure, namely tunica intima, tunica media, and tunica adventitia. The tunica intima is the innermost layer of the coronary arteries that is directly related to the incidence of atherosclerosis. This is because the accumulation of LDL until the formation of oxidized LDL causes endothelial cell activation and expression of many proinflammatory factors that stimulate the formation of atherosclerotic lesions.

\section{References}

1. B.R. Kwak, M. Back, M.L. Bochaton-Piallat, et al.Biomechanical factors in atherosclerosis: mechanisms and clinical implications Eur Heart J. 2014; 35: 30133020 CrossRefView Record in ScopusGoogle Scholar 
2. A.P. Burke, F.D. Kolodgie, A. Farb, et al .Healed plaque ruptures and sudden coronary death: evidence that subclinical rupture has a role in plaque progression Circulation. 2001; 103: 934-940 View Record in ScopusGoogle Scholar

3. E. Falk, M. Nakano, J.F. Bentzon, A.V. Finn, R. Virmani Update on acute coronary syndromes: the pathologists' view Eur Heart J. 2013; 34: 719-728 CrossRefView Record in ScopusGoogle Scholar

4. F.J. Gijsen, J.J. Wentzel, A. Thury, et al.Strain distribution over plaques in human coronary arteries relates to shear stress Am J Physiol Heart Circ Physiol, 2008; 295: H1608-H1614 CrossRefView Record in ScopusGoogle Scholar

5. G. Manoharan, A. Ntalianis, O. Muller, et al. Severity of coronary arterial stenoses responsible for acute coronary syndromes Am J Cardiol, 2009; 103: 1183-1188
ArticleDownloadPDFView Record in ScopusGoogle Scholar

6. G.W. Stone, A. Maehara, A.J. Lansky, et al.A prospective natural-history study of coronary atherosclerosis N Engl J Med. 2011; 364: 226-235 CrossRefView Record in ScopusGoogle Scholar

7. Hinsberg VWM, editor. Physiology of Boold Vessels. In: Krams R, Bäck M, editors.The ESC Textbook of Vascular Biology. Oxford, UK. $2017 ; 18$

8. Hansson GK. Libby, P. Inflammation and Plaque Vulnerability. Journal of Internal Medicine. 2015

9. Ibanez B. ESC Guidelines for the management of acute myocardial infraction in patients presenting with ST-segment elevation. 2018. Ehj. Available at: doi:10.1093/eurheartj/ehx393. 2017; 119177. 